\title{
Melanocitoma meníngeo del ángulo pontocerebeloso: ¿Un tumor benigno?
}

\author{
J. González-Tortosa; *B. Ferri-Ñíguez y J. Ros de San Pedro
}

Servicios de Neurocirugía y de *Anatomía Patológica. Hospital Universitario Virgen de la Arrixaca. Murcia.

\section{Resumen}

Se presenta un paciente con un raro melanocitoma meníngeo del ángulo pontocerebeloso que, tras su extirpación quirúrgica radical, evolucionó en el plazo de un año hacia una melanomatosis meníngea fulminante. Se realiza una revisión bibliográfica en busca de las claves para hacer una aproximación diagnóstica preoperatoria de este tipo de tumor y obtener información sobre su tratamiento y manejo postoperatorio.

PALABRAS CLAVE: Melanocitoma meníngeo. Ángulo pontocerebeloso. Melanocitosis meníngea. Tratamiento. Evolución. Pronóstico

Cerebellopontine angle meningeal melanocytoma: a benign tumor?

\section{Sumary}

We report a case of a rare meningeal melanocytoma in the cerebellopontine angle. One year after tumor gross total removal, the patient suffered a sudden and devastating meningeal melanomatosis. The relevant literature is reviewed looking for the keys to establish preoperative diagnosis and to obtain information about its treatment and postsurgical management.

KEY WORDS: Meningeal melanocitoma. Cerebellopontine angle. Meningeal melanomatosis. Treatement. Postsurgical evolution. Prognosis

\section{Introducción}

El melanocitoma es un tumor que se origina en los melanocitos de la leptomeninge y, desde su identificación en 1972 por Limas y $\mathrm{Tio}^{23}$, se han publicado casos esporádicos a nivel del ángulo pontocerebeloso ${ }^{13,17,18,32}$, donde puede ser confundido con otras lesiones melanocí-

Recibido: 15-12-07. Aceptado: 26-10-08 ticas, como el schwannoma melanocítico, el meningioma melanocítico o el melanoma primario. En principio, el melanocitoma está considerado como una lesión de curso prolongado y benigna. Sin embargo, se ha ido acumulando información bibliográfica que pone de manifiesto una incidencia preocupante de recidivas tras extirpaciones quirúrgicas radicales ${ }^{22,33,34}$, así como diseminaciones a lo largo del neuroaxis ${ }^{21,37}$ e, incluso, metástasis viscerales ${ }^{42}$, lo que pone entredicho la benignidad de la lesión ${ }^{29}$ y da relevancia al diagnóstico diferencial con otras lesiones benignas más frecuentes en esta zona, en especial con el schwannoma del acústico o el meningioma, ya que condiciona y plantea dudas sobre el manejo posquirúrgico de estos pacientes.

\section{Caso clínico}

Varón de 56 años con hipoacusia derecha de 7 meses de evolución, acúfenos y episodios de inestabilidad transitoria, con desequilibrios hacia uno y otro lado, que fueron empeorando de manera progresiva hasta precisar apoyo en la marcha en ocasiones.

En la exploración clínica solo se constató una hipoacusia moderada en el oído derecho (OD), con maniobra de Weber lateralizada hacia la derecha. Con excepción de la inestabilidad en la marcha, el resto de la exploración neurológica fue normal, incluido el fondo ocular. No presentaba manchas melánicas en la piel, ni en la conjuntiva ocular, ni lesiones pigmentadas en el fondo ocular.

\section{Pruebas complementarias}

Audiometría: hipoacusia neurosensorial en OD, con caída de agudos hasta los $70 \mathrm{db}$. Potenciales evocados auditivos: Solo se identifica la onda I en OD, que tiene una latencia normal. TAC: Masa homogénea, hiperdensa, en ángulo pontocerebeloso derecho, de $5 \mathrm{~cm}$. de diámetro, que comprime hemisferio cerebeloso, donde provoca edema perilesional, desplazando y colapsando el cuarto ventrículo sin provocar hidrocefalia. En la RM, el tumor es hiperintenso en T1, isointenso en T2 y tiene una captación de contraste enmascarada por la propia intensidad de la 


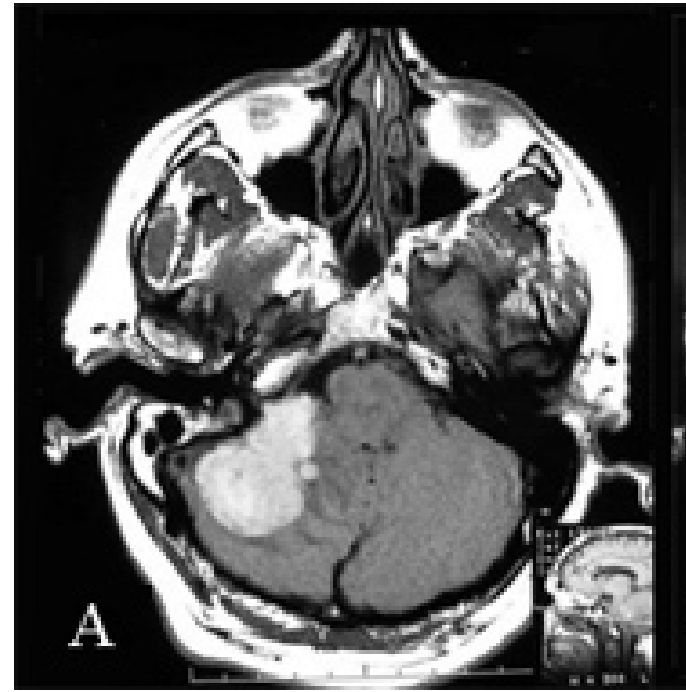

lesión (Figuras 1 A y B).

\section{Intervención quirúrgica}

Se realizó una craneotomía paramastoidea derecha. La duramadre cerebelosa presentaba una coloración azul oscura homogénea, recordando su aspecto al de los hematomas subdurales crónicos supratentoriales. Sin embargo, al abrirla, no se observó nada anormal en el espacio subdural, ni en la superficie del cerebelo. En el ángulo pontocerebeloso existía, lateralmente al tumor, un quiste aracnoideo de pared amarillo-verdosa. El tumor presentaba una coloración pardo oscura, con escasa consistencia, capsular por zonas, sangrando en sábana de manera abundante durante toda la operación. Se pudo separar bien del cerebelo, tronco cerebral y nervio estato-acústico. El

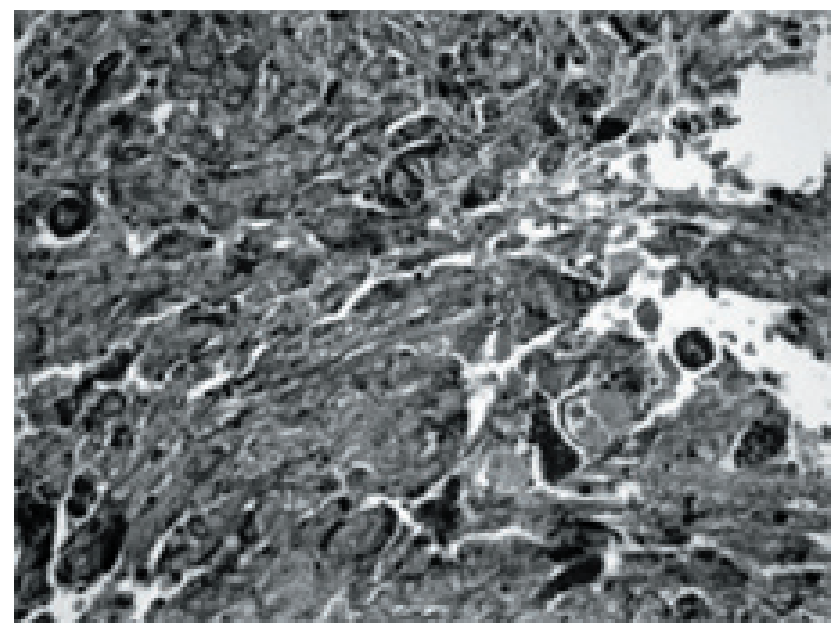

Figura 2. Proliferación de células ovoideas o redondas, sin atipias, con nucleolo poco llamativo, rodeadas por acúmulos de melanófagos y vasos capilares.
Figura 1. RM inicial de cráneo. A. RM T1 sin contraste: Tumor hiperintenso del ángulo pontocerebeloso derecho. $B$. RM T2: tumor isointenso que comprime y desplaza el hemisferio cerebeloso, donde provoca edema perilesional adyacente. El tumor está en intimo contacto con la cara posterior del peñasco, pero no agranda ni erosiona el CAI. Existe un acumulo de líquido cefalorraquideo lateral al tumor y una delgada capa de espacio aracnoideo que lo separa del tronco cerebral y cerebelo.

conducto auditivo interno tenía una morfología normal y no estaba invadido por el tumor. La neoplasia se encontraba adherida, a modo de base de implantación, en la duramadre de la cara posterior del peñasco, lateral y caudal al meato acústico interno. La extirpación fue completa, fulgurándose con electro-coagulación la base de implantación hasta dejar el hueso al descubierto.

\section{Examen histológico}

En el estudio histológico del tumor extirpado se observó que, la totalidad de la neoplasia, estaba constituida por una proliferación sólida de células sin pleomorfismo, relativamente monomorfas y de aspecto epitelioide, fusocelular o poligonal, dispuestas en nidos y con tendencia al "arremolinamiento". Los núcleos eran redondos u ovalados, con nucleolo poco llamativo y los citoplasmas estaban cargados intensamente de pigmento granular de color pardo-oscuro. En la vecindad, también se identificó gran cantidad de melanófagos (Figura 2). No se identificaron figuras de mitosis ni tampoco áreas de necrosis o hemorragia. El índice de proliferación celular (ki-67) fue inferior al $2 \%$.

El pigmento citoplasmático de las células proliferadas y el de los melanófagos, mostró intensa positividad para Masson-Fontana y de forma focal se identificó la presencia de pigmento férrico con tinción de Perls. Las células también mostraban intensa positividad para proteína S-100, HMB-45, Melan-A y MITF - Factor de Transcripción de la Microftalmia - (Figura 3), así como positividad focal para Vimentina y NSE. Las tinciones de Citoqueratina AE1AE3, PAGF, EMA y Leu-7 fueron negativas (Fig. 4). Estos resultados son consistentes con el diagnóstico de melanocitoma meníngeo. 


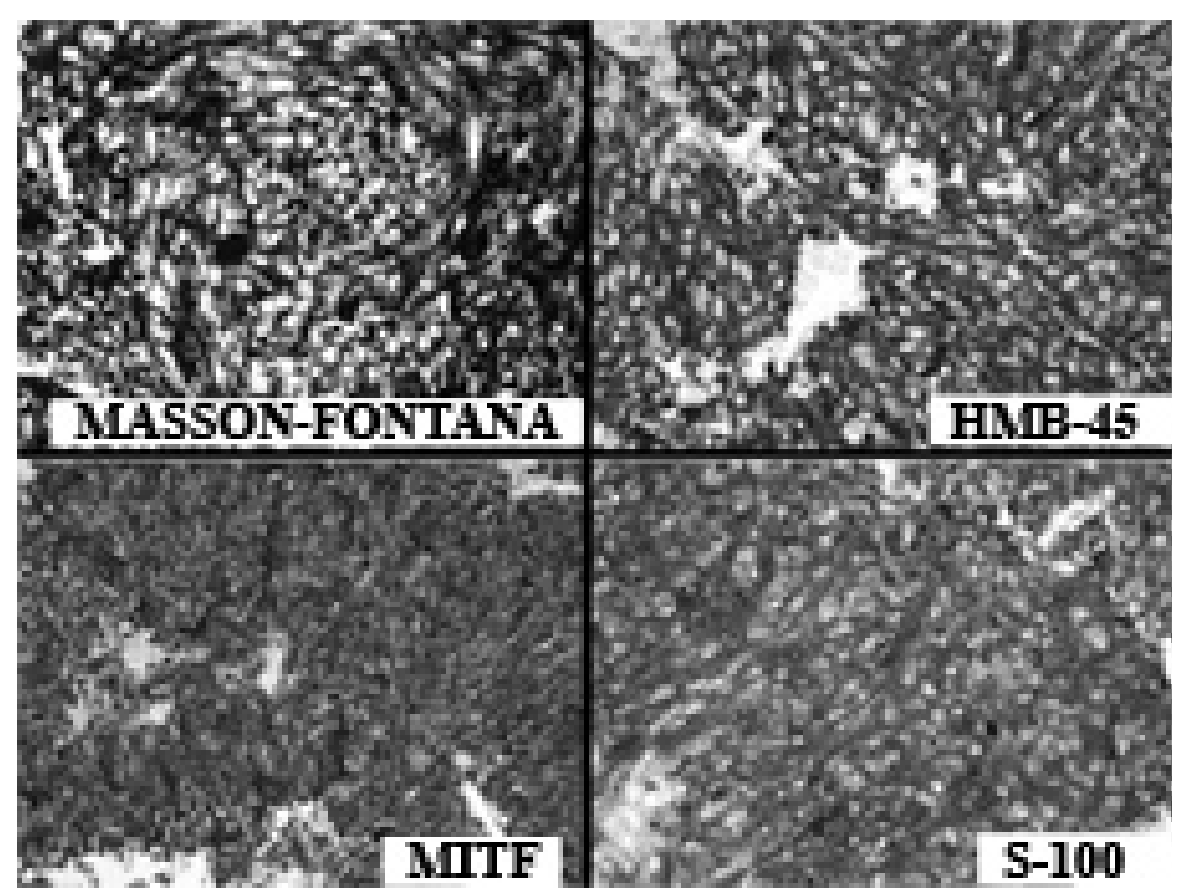

2009; 20: $372-380$

\section{Evolución}

El postoperatorio cursó sin incidencias. El paciente fue revisado en consulta externa a los seis y once meses después de la intervención quirúrgica, habiendo tenido un curso favorable con desaparición de los acúfenos y de la inestabilidad en la marcha, aunque persistía una ligera hipoacusia neurosensorial derecha. Ambas RM de control en esas fechas, mostraron la ausencia completa de tumor.
Tres meses más tarde de la última revisión, el paciente ingresó en otro hospital por un cuadro de una semana de evolución, consistente en somnolencia, agitación nocturna, afasia mixta, nistagmus horizontal y vertical, junto con paresia e hiporreflexia de miembros inferiores. En el estudio de RM se evidenció una lesión extra-axial en ángulo pontocerebeloso derecho de $2 \mathrm{~cm}$ de diámetro, con las características de la lesión original en T1 y T2, así como un realce y engrosamiento leptomeníngeo difuso (Figuras $5 \mathrm{~A}$

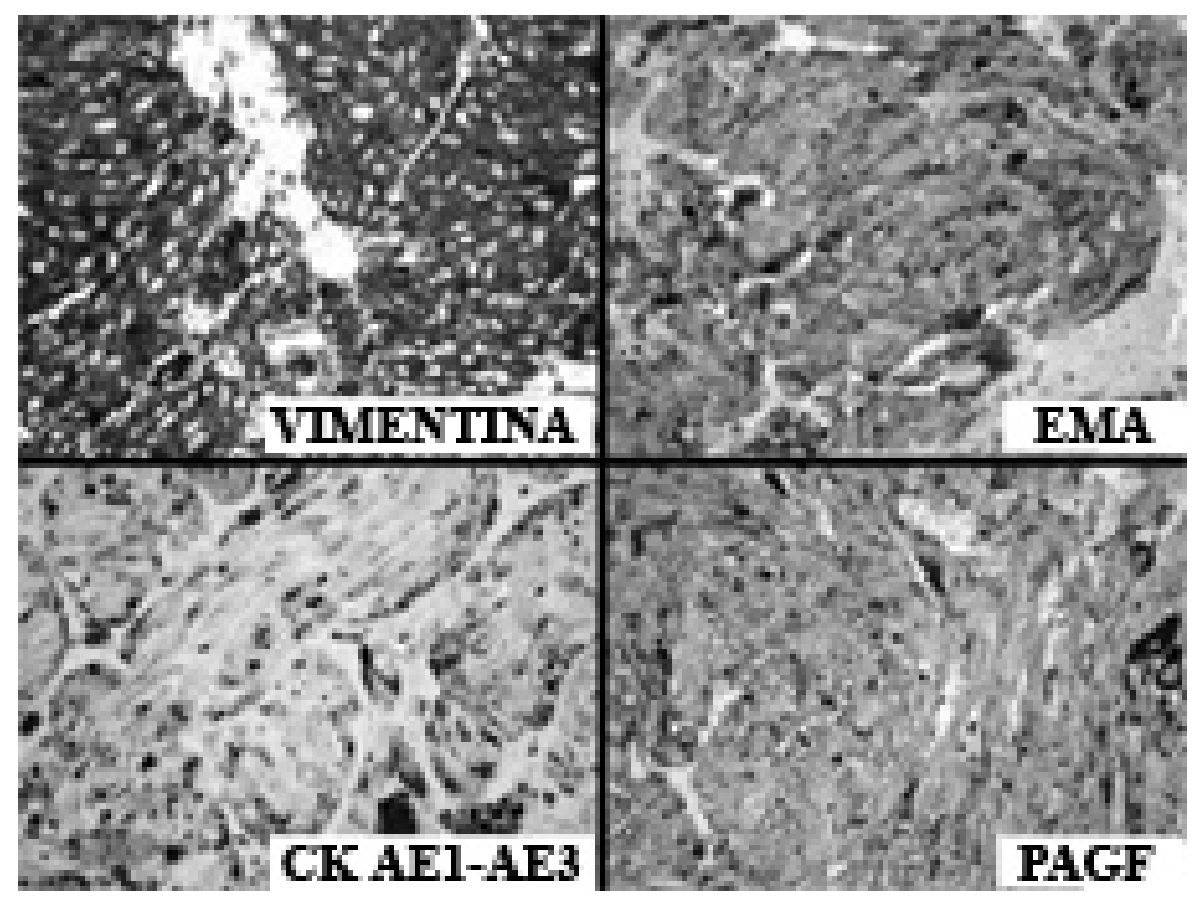

Figura 3. Tanto las células neoplásicas como los melanófagos contienen gran cantidad de pigmento melánico positivo con la tinción de Masson-Fontana (imagen superior izquierda). Obsérvese la intensa inmunotinción de las células para marcadores melanociticos (resto de imágenes).
Figura 4. En la imagen superior izquierda puede observarse la intensa tinción para Vimentina y la negatividad para Antígeno de Membrana Epitelial, Citoqueratina AE1-AE3 y Proteina Acida GLiofibrilar en el resto de imágenes. 


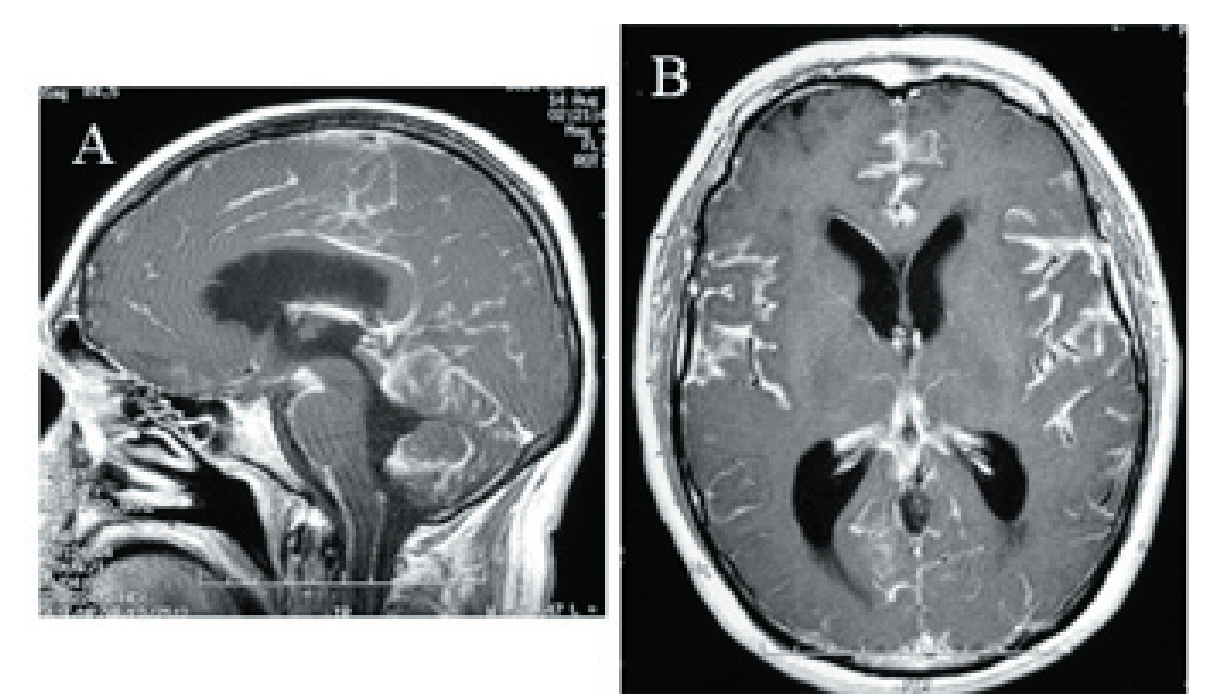

2009 20: $372-380$
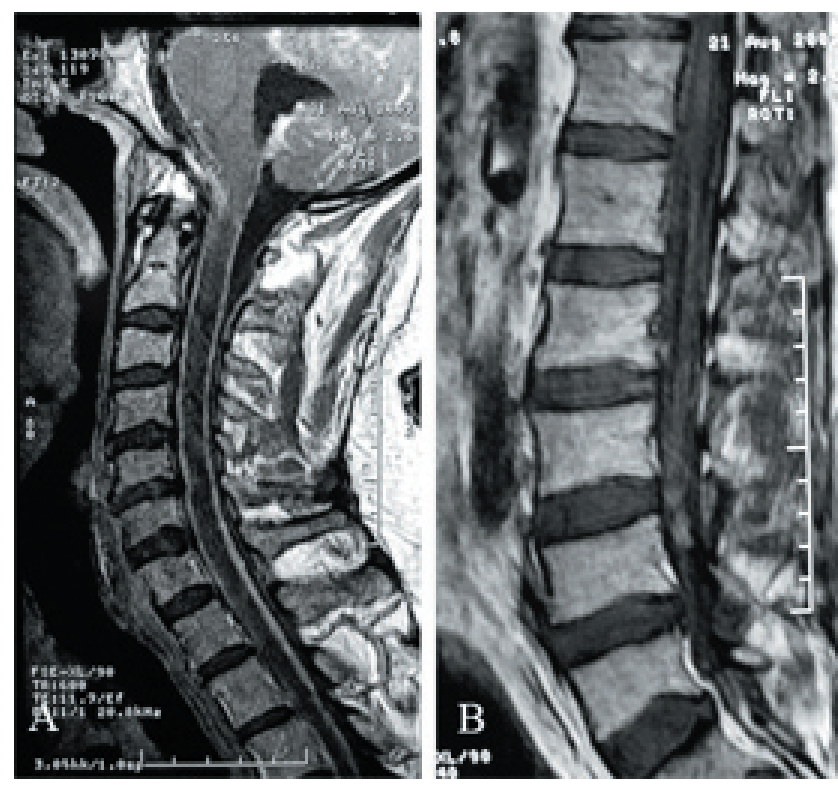

Figura 6. $A$ : RM tras contraste: realce y engrosamiento de las meninges cervicales. B: RM T1: nódulos tumorales hiperintensos en cauda equina.

y B) e hidrocefalia moderada. En las secuencias FLAIR, existían pequeños focos hiperintensos en sustancia blanca supratentorial, junto a los surcos de la convexidad cerebral. En la RM de raquis (Figuras 6 A y B), se apreciaba un engrosamiento difuso y realce meníngeo cervical, dorsal y lumbar, con algún engrosamiento más nodular en alguna zona, en especial en cauda equina (Figura 6B).

La punción lumbar mostró un líquido xantrocrómico positivo para células de melanoma.

El paciente recibió tratamiento con esteroides y una primera dosis de quimioterapia con Fotemustina, pero sufrió un deterioro neurológico progresivo y falleció unos días más tarde, cuando se cumplían catorce meses desde la intervención quirúrgica.

\section{Discusión}

Los melanocitos de la capa basal de la epidermis y de las leptomeninges, tienen un origen común a partir de elementos que derivan de la cresta neural, durante el desarrollo temprano del embrión. A nivel de las leptomeninges son más abundantes en la base de cráneo, en especial en fosa posterior, a nivel de la superficie ventro-lateral de la médula oblongata ${ }^{14}$ y en región cervical. Limas y Tio en $1972^{23}$, mediante microscopía electrónica, identificaron por primera vez el origen de estos tumores a partir de estos melanocitos y no de los fibroblastos meníngeos, como previamente se pensaba, acuñando el término de melanocitoma meníngeo para diferenciarlo del meningioma melanocítico, que tiene gran contenido de melanina. Posteriormente se han desarrollado técnicas de inmuno-tinción que contribuyen a diferenciarlos.

La clasificación de los tumores del sistema nervioso central de la World Health Organization (WHO) ${ }^{25}$, incluye el melanocitoma meníngeo entre las lesiones melanocíticas primarias de las meninges. Es un tumor benigno que tiene en el melanoma, bien focal o difuso (melanomatosis meníngea), su equivalente maligno.

\section{Localización}

El melanocitoma suele ser una lesión extra-axial ${ }^{1}$ con predilección por fosa posterior, cavum de $\mathrm{Meckel}^{6}$, agujero magno ${ }^{40}$, regiones cervical ${ }^{15}$ y torácica ${ }^{26}$ de columna vertebral y, con menos frecuencia, lumbar ${ }^{42}$. Se han publicado casos esporádicos a nivel lóbulo temporal ${ }^{2,19}$, región frontal $^{20}$, seno cavernoso ${ }^{12}$, plano esfenoidal $^{7}$, región pineal ${ }^{10} \mathrm{y}$ convexidad parietal $^{3}$, así como lesiones intramedulares ${ }^{33,39}$ e intraorbitarias ${ }^{11}$. Solo hemos encontrado nueve casos 
publicados en el ángulo pontocerebeloso, incluido el nues$\operatorname{tro}^{8,13,17,18,21,32}$.

\section{Características clínicas}

En conjunto, la edad media de los pacientes es de 44 años (rango 9-75) 3 $^{33}$ con una duración de los síntomas de cuatro semanas a 14 años ${ }^{7}$. Son tumores de crecimiento lento, por lo que suelen tener un tamaño considerable en el momento del diagnóstico ${ }^{2}$.

En el ángulo pontocerebeloso provocan los síntomas congruentes con esta localización. Es de destacar que suelen provocar pérdida auditiva neurosensorial ${ }^{8,17}$, como en nuestro caso, por lo que pueden ser confundidos con otros tumores más frecuentes de esta zona, en especial el schwannoma acústico, pero el conducto auditivo interno no está agrandado ni erosionado. Esto es importante, porque permite, al tratarse de un tumor grande, dar mas posibilidades al paciente de que el nervio facial sea respetado durante la intervención quirúrgica.

\section{Diagnóstico radiológico}

En la TAC los melanocitomas aparecen constantemente como tumores extra-axiales, bien delimitados, isodensos o ligeramente hiperdensos, con una captación homogénea del contraste, similar a los meningiomas ${ }^{6}$. En la RM, aunque pueden ser isointensos en $\mathrm{T} 1^{11,12,24,27,33}$, con más frecuencia son hiperintensos ${ }^{1,6,8,9,10,20,26,40}$, en cuyo caso ayuda a diferenciarlos de los meningiomas, que suelen ser isointensos, y de los schwannomas, que casi siempre se muestran

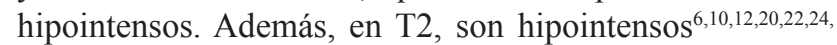
${ }^{26,27,40}$ con pocas excepciones ${ }^{9,11,33}$, incluido nuestro paciente. El comportamiento del tumor en la RM está relacionado con la cantidad de melanina presente, de manera que cuanta más abundancia de melanina, mas se acortan los tiempos de relajación en $\mathrm{T} 1$ y $\mathrm{T}^{6}$.

\section{Datos quirúrgicos}

En el campo quirúrgico aparecen como tumores bien encapsulados, con una coloración oscura que sugiere el contenido alto de melanina en el tumor. El quiste aracnoideo que existía lateral al tumor en nuestro paciente, es el habitual que se encuentra en los tumores grandes del acústico. Su base de implantación se encontraba en la dura madre de la cara posterior del peñasco, sin relación con el meato acústico interno, al igual que en otros casos publi$\operatorname{cados}^{17,18}$. En ocasiones puede estar adherido a los pares craneales o al tronco cerebral ${ }^{17,29}$, así como sangrar de manera abundante ${ }^{8}$, hasta el punto de dificultar su extirpación completa. En nuestro caso, el abundante sangrado en sábana no impidió la exéresis radical de la lesión.
La coloración azul oscura que observamos en la paquimeninge adyacente al hemisferio cerebeloso derecho en nuestro paciente, aunque no se analizó desde el punto de vista histológico, nos sugiere la posibilidad de que existiera una melanocitosis meníngea, adicional a la presencia del tumor.

\section{Diagnóstico histológico}

Desde el punto de vista histológico, los melanocitomas plantean problemas de diagnóstico diferencial con otros tumores que pueden contener melanina. Por la localización a nivel del ángulo pontocerebeloso en nuestro paciente, es necesario distinguirlo del schwanoma melanocítico y del meningioma melanocítico. Desde el punto de vista pronóstico, es esencial distinguirlos de los melanomas.

En acuerdo con la literatura, las características histológicas del melanocitoma meníngeo recuerdan mucho al meningioma melanocítico, con el que se ha confundido durante años. Consiste en haces y nidos de células fusiformes o epiteloides, con núcleo vesicular redondo $\mathrm{u}$ oval, nucleolo variable y, a veces, prominente, con abundante melanina en el citoplasma y en los melanófogos acompañantes. El estroma es fibrovascular y las células tienen tendencia a formar remolinos. Una característica muy importante, es que tienen una actividad mitótica o índice de proliferación muy bajo, y deben de estar ausentes las atípias celulares, la necrosis, hemorragia o invasión del parénquima nervioso.

El análisis inmunohistoquímico es indispensable para diferenciar el melanocitoma meníngeo de otras lesiones pigmentadas similares. Así, el melanocitoma meníngeo, se caracteriza por tener una reacción positiva a proteína S-100 y Vimentina y a los anticuerpos antimelanoma (HMB45), MITF y Melan-A, al tiempo que tienen una reacción negativa para el antígeno de membrana epitelial (EMA). El meningioma melanocítico se caracteriza por coexpresar EMA y Vimentina y es negativo para la proteína S-100 y para el resto de marcadores melanocíticos (HMB-45, Melan-A y MITF). De igual manera, la positividad para estos últimos marcadores de estirpe melanocítica ayuda a excluir el diagnóstico de schwannoma pigmentado.

Mas difícil es diferenciar, a nivel inmunohistoquímico, el melanocitoma del melanoma ${ }^{29}$, ya que ambos comparten la positividad para HMB-45, Melan-A, MITF y proteína S-100 y en ocasiones también expresan Vimentina. La principal diferencia viene dada por la ausencia de signos de malignidad histológica como el pleomorfismo celular y atipias nucleares, la presencia de mitosis abundantes -Ki-67 no superior al 2\%- necrosis y hemorragia. El diagnóstico diferencial entre un melanoma primario o metastático, no es posible realizarlo desde el punto de vista histológico, por lo que es necesario realizar un examen clínico exhaustivo 
para descartar la existencia de un tumor primitivo cutáneo, en el fondo ocular y mucosas.

La microscopía electrónica de las células tumorales demuestra su diferenciación melanocítica con presencia de procesos dendríticos citoplasmáticos y melanosomas en varios estadios de maduración. Estos hallazgos son determinantes para diferenciar el melanocitoma meníngeo del meningioma melanocítico, cuando encuentra melanosomas en el interior de las células y ausencia de desmosomas. La lámina basal alrededor de las células tumorales no está bien desarrollada, lo que lo diferencia también del schwannoma melanótico ${ }^{10}$.

\section{Evolución y pronóstico}

Kurita y cols. ${ }^{22}$ revisaron la evolución de 14 pacientes con melanocitoma meníngeo de diferente localización; cinco pacientes $(37,7 \%)$ sufrieron recurrencia local entre los 6 y 60 meses tras la intervención quirúrgica, pese a que en tres de ellos la resección había sido total. Otros tres pacientes con resección parcial, recibieron radioterapia fraccionada que no impidió la recidiva. El índice de recidiva postoperatoria global, se calculó en el $50,9 \%$ a los cuatro años. Rades y cols. ${ }^{33}$ entre 46 pacientes con un periodo de evolución postoperatoria de 4 años, encontraron recidivas en el $31 \%$ de las extirpaciones completas, en el $45 \%$ de las extirpaciones parciales seguidas de radioterapia, y en el $100 \%$ de los casos en los que sólo se había hecho una resección parcial del tumor. La supervivencia global fue de $74 \%$ a los 5 años. Tuhan y cols. ${ }^{39}$, no encontraron recidivas en 9 resecciones completas, pero si en 6 de 10 pacientes con resecciones parciales, cinco de ellos habiendo recibido radioterapia adicional. En la serie de 49 pacientes con melanocitomas espinales de Rades y Schild ${ }^{34}$, el control local del tumor a los 5 años de evolución, fue de $78 \%$ con extirpación completa y $22 \%$ con extirpación parcial $(\mathrm{p}<$ 0,001 ) y una supervivencia de $83 \%$ y $40 \%$ respectivamente $(p=0,011)$. La extirpación parcial obtuvo un mejor control local del tumor, a los 5 años, con radioterapia que sin ella $(59 \%$ vs $22 \%, p=0,029)$ y una tendencia más favorable en cuanto a la supervivencia ( $85 \%$ vs $40 \%, \mathrm{p}=0,10)$.

Brat y col. ${ }^{4}$ estudiaron 17 casos de melanocitomas histológicamente típicos, en cuanto a la ausencia absoluta de signos de malignidad y MIB- 1 de $<2 \%$; ninguno recidivó tras la extirpación quirúrgica. En 13 melanomas primarios malignos, con un promedio MIB-1 del 8,1\%, presentaron recidiva tras la cirugía el $61 \%$, con un periodo de supervivencia promedio de 14 meses. Uno de los aspectos interesantes de este trabajo es que, tres pacientes presentaban grados histológicamente intermedios, con MIB-1 entre el 1-4\%, de manera que no pudieron ser clasificados como melanocitoma o melanoma. De los tres, uno se perdió para la evolución, pero de los otros dos, uno recidivó a los
17 meses y el otro murió tras la cirugía. Es decir, existen grados intermedios, a veces de difícil interpretación, que complican el diagnóstico y la valoración pronóstica del paciente $^{36}$.

Se ha demostrado la trasformación maligna del melanocitoma tanto en la recidiva local ${ }^{37,41,42}$, como en diseminación leptomeningea ${ }^{21,37}$, bien en forma de metástasis a distancia dentro del neuroeje $\mathrm{e}^{5,33}$ o como melanomatosis meníngea ${ }^{41}$. Incluso se han publicado metástasis fuera del $\mathrm{SNC}^{42}$. La carcinomatosis meníngea, en la que el melanoma es la tercera causa más frecuente ${ }^{16}$, el LCR es anormal en más del $95 \%$ de los casos, siendo la citología positiva para células tumorales hasta en el $81 \%$ de los enfermos, si se busca de forma repetida. La RNM es muy sensible para mostrar el realce meníngeo tumoral ${ }^{16,30,31}$, como sucedió en nuestro enfermo, aunque es compartido por otras patologías $^{35}$. La supervivencia en estos casos es de menos de 2 meses sin tratamiento y de 5,8 meses con radiación y quimioterapia $^{16}$.

Aunque muchos melanocitomas pueden sobrevivir durante muchos años, el alto porcentaje de recidivas incluso con resecciones totales, el índice de supervivencia y las transformaciones malignas publicadas, ha llevado a algún autor a cuestionar la benignidad que la WHO adjudica a estas lesiones ${ }^{28}$, lo que apoya la evolución sufrida por nuestro paciente.

\section{Tratamiento}

Existe un consenso en que, en este tipo de tumor, hay que intentar siempre la resección completa como mejor modalidad de tratamiento, ya que es la que proporciona el menor índice de recidivas y la mayor supervivencia.

La valoración del beneficio de la radioterapia en estos raros tumores, así como la dosis idónea, está limitada por el insuficiente número de pacientes en las cohortes, el carácter retrospectivo de los estudios y la ausencia de una revisión patológica centralizada.

En los casos de extirpaciones parciales parece que existe, como se ha comentado antes, una tendencia a mejores resultados en cuanto a recidiva y supervivencia de estos pacientes si se completa el tratamiento con radioterapia local y, de hecho, la recomiendan todos los autores.

Rades y Schild ${ }^{34}$, establecieron en los melanocitomas espinales, la dosis de 50-52,2 Gy como la que mejor resultado obtiene en cuanto al control local del tumor, con correcciones según la longitud de médula irradiada. En las lesiones intracraneales, sin embargo, no existe información suficiente como para establecer la dosis de irradiación más adecuada.

No se tiene experiencia con la radiocirugía. Kurita y cols. ${ }^{22}$ han publicado un caso de melanocitoma del cavum de Meckel extirpado de forma incompleta que, tras su trata- 
miento en gamma knife con una dosis marginal de $25 \mathrm{~Gy}$, el tumor disminuyó su volumen residual a los 3 años. Uozomi y cols. ${ }^{41}$, tras aplicarla en una recidiva local, el tumor tuvo un crecimiento agresivo.

Rades y Schild ${ }^{34}$, recomiendan la radioterapia local incluso en los enfermos en los que la resección del melanocitoma espinal haya sido completa, basándose en la tasa de recidiva $(22 \%)$ que tienen estos tumores a los cinco años. Dado que el índice de recidiva local de los melanocitomas, sin especificar localización es, como se ha comentado antes, del 31-37,7\%, con casos de trasformación maligna y diseminación leptomeníngea publicados, incluido nuestro paciente, nosotros también nos inclinamos por la radioterapia complementaria, incluso cuando la extirpación del tumor haya sido radical.

Desconocemos el valor de realizar citologías posquirúrgicas del LCR en esta patología pero, sin duda, si en algún caso fuera positiva para células tumorales, cambiaría por completo el manejo posquirúrgico que se suele realizar con estos pacientes.

\section{Conclusiones}

El melanocitoma es un tumor de histología benigna, para el que existe consenso en que el mejor tratamiento es la resección quirúrgica total, reservando la radioterapia para los casos de extirpación parcial. Sin embargo, tiene un porcentaje de recidiva local nada despreciable, incluso tras la resección quirúrgica completa, y conserva un potencial de transformación maligna y de diseminación por el neuroeje que no es acorde con su clasificación histológica y pone en entredicho su carácter clínico benigno.

La rareza de esta neoplasia hace necesario la puesta en marcha de un estudio centralizado multi-institucional, que incluya la supervisión histológica, para poder llegar a conclusiones estadísticamente significativas en cuanto a la mejor pauta de tratamiento.

\section{Bibliografía}

1. Ahluwalia, S., Ashkan, K., Casey, AT.: Meningeal melanocytoma: clinical features and review of the literature. Br. J. Neurosurg. 2003; 17: 347-351.

2. Aimar, E., Debernardi, A., Tancioni, F., et al.: Meningeal melanocytoma of the temporal lobe. An uncommon tumor in an unusual location. Case report. J. Neurosurg. Sci. 2003;47: 211-214.

3. Baseoglu, K., Knobbe, C.B., Reifenberger, G., Steiger, H.J., Stummeer, W.: Supratentorial meningeal melanocytoma mimicking a convexity meningioma. Acta Neurochir. (Wien) 2006; 148: 485-490.

4. Brat, D.J., Giannini, C., Scheithauer, B.W., Burger, P.C.: Primary melanocytic neoplasms of the central nervous systems. Am. J. Surg. Pathol. 1999; 23: 745-754.

5. Bydon, A., Gutierrez, J.A., Mahmood, A.: Meningeal melanocytoma: an aggressive course for a benign tumor. J. Neurooncol. 2003; 64: 259-263.

6. Chen, C.J., Hsu, Y.I., Ho, Y.S., Hsu, Y.H., Wang, L.J., Wong, Y.C.: Intracraneal meningeal melanocytoma: CT and MRI. Neuroradiology 1997; 39: 811-814.

7. Chow, M., Clarke, D.B., Maloney, W.J., Sangalang, V.: Meningeal melanocytoma of the planum sphenoidale. Case report and review of the literature. J. Neurosurg. 2001; 94: 841-845.

8. Clarke, D.B., Leblanc, R., Bertrand, G., Quartey, G.R., Snipes, G.J.: Meningeal melanocytoma. Report of a case and a historical comparison. J. Neurosurg. 1998; 88: 116-21.

9. Czarnecki, E.J., Silbergleit, R., Gutierrez, J.A.: MR of the spinal meningeal melanocytoma. AJNR 1997; 18: 180182.

10. Czirják, S., Vitanovic, D., Slowik, F., Magyar, A.: Primary meningeal melanocytoma of the pineal region. J. Neurosurg. 2000; 92: 461-465.

11. de Tella, O.I.Jr., Agner, C., Aguiar, P.H., Herculano, M.A., Prandini, M.N., Stavile, J.N.: Aggressive management of orbital meningeal melanocytoma. Acta Neurochir. (Wien) 2003; 145: 1121-1126.

12. Faro, S.H., Koenigsberg, R.A., Turtz, A.E., et al.: Melanocytoma of the cavernous sinus: CT and MR findings. AJNR 1996; 17: 1087-1090.

13. Gardiman, M., Altavilla, G., Marchioro, L., Boscolo, L., Alessio, L., Piazza, M.: Meningeal melanocytoma: a rare lesion of the central nervous system. Tumori. 1996; 82: 494496.

14. Goldgeier, M.H., Klein, L.E., Klein-Angerer, S., Moellmann, G., Nordlund, J.J.: The distribution of melanocytes in the leptomeninges of the human brain. J. Invest. Dermatol. 1984; 82: 235-238.

15. Gonçalves, J., Díaz, P., Subhi-Issa, I., Maillo, A., Blanco, A.: Spinal meningeal melanocytoma simulating neurinoma: case report. Neurocirugia 2002; 13: 393-396.

16. Greenberb, M.S.: Carcinomatous meningitis. En Greenberg MS (ed). Handbook of Neurosurgery. Fifth edition. Stuttgart (Germany). Thieme International. 2001; pp: 469-4.

17. Gupta, A., Ahmad, F.U., Sharma, M.C., Garg, A., Mehta, V.S.: Cerebelopontine angle meningeal melanocytoma: A rare tumor in an uncommun location. J. Neurosurg. 2007; 106: 1094-1097.

18. Hamasaki, O., Nakahara, T., Sakamoto, S., Kutsuna, M., Sakoda, K.: Intracranial meningeal melanocytoma. Neurol. Med. Chir. (Tokyo) 2002; 42: 504-509.

19. Kang, S.H., Yoo, D.S., Cho, K.S., et al.: Coexisting intracranial meningeal melanocytoma, dermoid tumor, and Candy-Walier cyst in a patient with neurocutaneous melanosis. J. Neurosurg. 2006; 104: 444-447.

20. Kawaguchi, T., Kawano, T., Kazekawa, K., et al: 
Meningeal melanocytoma in the left frontal region. Brain Tumor Pathol. 1998 ; 15: 58-62.

21. Koch, H.J., Roeber, S., Zimmermann, U.W., et al.: Spinal and cerebral leptomeningeal seeding from a melanocytoma of the cerebello-pontine angle. Wien Med. Wochenschr. 2005; 155: 360-364.

22. Kurita, H., Segawa, H., Shin, M., et al.: Radiosurgery of meningeal melanocytoma. J. Neurooncol. 2000; 46: 57-61.

23. Limas, C., Tio, F.O.: Meningeal melanocytoma ("melanotic meningioma"). Its menlanocytic origin as revealed by electron microscopy. Cancer 1972; 30: 1286-1294.

24. Litofsky, N.S., Zee, C.S., Breeze, R.E.: Meningeal melanocytoma: diagnostic criteria for a rare lesion. Neurosurgery $1992 ; 31: 945-948$.

25. Louis, D.N., Ohgaki, H., Wiestle,r O.D., et al.: The 2007 WHO classification of tumours of the central nervous system. Acta Neuropathol. (Berl) 2007; 114: 97-109.

26. Matsumoto, S., Dang, Y., Sato, S., et al.: Spinal meningeal melanocytoma presenting with superficial siderosis of the central nervous system. J. Neurosurg. 1998; 88: 890-894.

27. Naul, L.G., Hise, J.H., Bauserman, S.C., et al.: CT and MR of meningeal melanocytoma. AJNR 1991; 12: 315-316.

28. O'Brien, D.F., Crooks, D., Mallucci, C., et al.: Meningeal melanocytoma. Childs Nerv. Syst. 2006: 22: 556-561.

29. O'Brien, T.F., Moran, M., Miller, J.H., Hensley, S.D.: Meningeal melanocytoma. An uncommon diagnostic pitfall in surgical neuropathology. Arch. Pathol. Lab. Med. 1995; 119: 542-546.

30. Painter, T.J., Chaljub, G., Sethi, R., Singh, H., Gelman, B.: Intracranial and intraspinal meningeal melanocytosis. AJNR 2000; 21: 1349-1353.

31. Pirini, M.G., Mascalchi, M., Salvi, F., et al.: Primary diffuse meningeal melanomatosis: radiologic-pathologic correlation. AJNR 2003; 24: 115-118.

32. Prabhu, S.S., Lynch, P.G., Keogh, A.J., Parekh, H.C.: Intracranial meningeal melanocytoma: a report of two cases and a review of the literature. Surg. Neurol. 1993; 40: 516521.

33. Rades, D., Heidenreich, F., Tatagiba, M., Brandis,

\section{Comentario al trabajo Melanocitoma meníngeo del ángulo pontocerebeloso: ¿Un tumor benigno? de Gonzá- lez-Tortosa y cols.}

He leido con gran interés el caso clínico presentado por González-Tortosa y cols.; en él se señalan las características clínicas, quirúrgicas, histopatológicas y pronósticas del caso presentado y se documenta de manera completa la experiencia recogida en la literatura en los pacientes con melanocitoma meníngeo. En éste en concreto, existe
A., Karstens, J.H.: Therapeutic options for meningeal melanocytoma. Case report. J. Neurosurg. (Suppl) 2001; 95: 225231.

34. Rades, D., Schild, S.E.: Dose-response relationship for fractionated irradiation in the treatment of spinal meningeal melanocytomas: a review of the literature. J. Neurooncol. 2006; 77: 311-314.

35. River, J., Schwartz, A., Gomoi, J.M., Soffer, D., Siegal, T.: Clinical significance of diffuse dural enhancement detected by magnetic resonance imaging. J. Neurosurg. 1996; 85: 777783 .

36. Rosai, J.: Neuromuscular system: central nervous system. En Rosai J (ed). Ackerman's Surgical Pathology. $9^{\text {th }}$ edition. St. Louis: Mosby, 2004; pp. 2585-2586.

37. Roser, F., Nskamura, M., Brandis, A., Hans, V., Vorkapic, P., Samii, M.: Transition from meningeal melanocytoma to primary cerebral melanoma. J. Neurosurg. 2004; 101: 528-531.

38. Saha, S., Meyer, M., Krementz, E.T., et al.: Prognostic evaluation of intracranial metastasis in malignant melanoma. Ann. Surg. Oncol. 1994; 1: 38-44.

39. Turhan, T., Oner, K., Yurtseven, T., Akalin, T., Ovul, I.: Spinal Meningeal melanocytoma. J. Neurosurg. (Spine) 2004; 100: $287-290$.

40. Uematsu, Y., Yukawa, S., Yokote, H., Itakura, T., Hayashi, S., Komai, N.: Meningeal melanocytoma: magnetic resonance imaging characteristics and pathological features. J. Neurosurg. 1992; 76: 705-709.

41. Uozumi, Y., Kawano, T., Kawaguchi, T., et al.: Malignant transformation of meningeal melanocytoma: a case report. Brain Tumor Pathol. 2003; 20: 21-25.

42. Wang, F., Li, X., Chen, L., Pu, X.: Malignant transformation of spinal meningeal melanocytoma. J. Neurosurg. 2007; 6: 451-454.

González-Tortosa, J.; Ferri-Ñ́guez, B.; Ros de San Pedro, J.: Melanocitoma meníngeo del ángulo pontocerebeloso: ¿Un tumor benigno? Neurocirugía 2009; 20: 372-380.

Correspondencia con los autores: tortosa@ctv.es la infrecuente evolución hacia una melanomatosis difusa, lo que le confiere tan mal pronóstico al paciente. Como los autores señalan oportunamente, sólo la coloración azulada de la meninge durante el acto quirúrgico parece poder relacionarse con esta evolución, dado que la RM preoperatoria no ofrecía realce ni engrosamiento de las meninges. 
Es fundamental constatar esos datos durante la inspección y descripción quirúrgica de las lesiones, aunque a veces ni estos puedan presagiarnos el pronóstico ${ }^{2}$, frente a la histopatología la cual se correlaciona en la mayor, que no siempre, parte de los casos ${ }^{1}$.

\section{Bibliografía}

1. Gil-Salú, J.L., Rodríguez-Peña, F., López-Escobar, M., Palomo, M.J.: Meduloblastoma de presentación extra-axial en el ángulo pontocerebeloso. Neurocirugía 2004; 15: 285-289.

2. Louis, D.N., Ohgaki, H., Wiestler, O.D., et al.: The 2007

J.L. Gil Salú

Cádiz
WHO classification of tumours of the central nervous system. Acta Neuropathol. (Berl) 2007; 114: 97-109. 